\title{
Extragenital bullous and hemorrhagic Lichen sclerosus in a 58-year-old woman - A case report
}

\author{
Stoyan Ivanov Pavlov ${ }^{1 *}$, Dimitra Petrova Nikiforova ${ }^{1}$, Silvi Vicheva Georgieva ${ }^{1}$ and Penka Liobenova Kolova-Peneva ${ }^{2}$ \\ ${ }^{1}$ Department of Dermatology and Venereology, University Hospital “St.Marina”, Medical University of Varna, Varna, Bulgaria \\ ${ }^{2}$ Department of Pathology, University Hospital “St.Marina”, Medical University of Varna, Varna, Bulgaria
}

\begin{abstract}
Lichen sclerosus (LS) is a rare, chronic, mucocutaneous disease, with a genital and extragenital manifestation. The etiology is not completely clarified, but there are suspicions for the role of genetic, autoimmune, infectious or hormonal factors.

We present a 58-year-old woman with five-year evolution of rare bullous-hemorrhagic type of Lichen sclerosus, engaging the trunk and limbs and verified by histopathological examination. The disease was characterized by the appearance of hemorrhagic bullae on the sclero-atrophic plaques. The lesions were locally treated with high-potency topical corticosteroid (Clobetasol propionate $0.05 \%$ cream). A solution with antiseptic action (Povidone-iodine $10 \%$ ) was administered for the erosion, caused by a rupture of the bulla. As a result, the erosion fully epithelized for a month. This case describes a rare type of Lichen sclerosus which responded favorably to the local treatment with corticosteroids and antiseptics.
\end{abstract}

\section{Introduction}

Lichen sclerosus (LS) a rare inflammatory disease, described for the first time of Hallopeau in 1887 [1]. The histopathology of the disorder was defined by Darier in 1892 [2].

Clinical manifestations are atrophic, white papules or plaques, located most frequently in anogenital region. The appearance of bullae on the atrophic plaques is very rarely observed, particularly in extragenital LS [3]. The disease affects both genders with female/male ration of $10: 1$ and is diagnosed in 5 to 6 decade of life. The frequency of extragenital location is reported to be $15-20 \%$ [2]. The etiology is an object of broad discussions and the role of genetic factors, autoimmunity, hormonal disturbances due to thyroid pathology and infections pathogens, like Borrelia burgdorferi and Hepatitis $\mathrm{C}$ virus have been investigated $[4,5]$. The standard treatment of disease is a local application of potent corticosteroids with disputable results.

\section{Clinical case description}

We present a 58-year-old woman, working in a factory for locks and metal mountings for furniture. She suffered from whitish papules and plaques, engaging the trunk and limbs with a duration of five years without other concomitant illnesses.

\section{Evolution of the disease}

The changes began with symmetrical redness and thickening of the skin of the abdomen, around the umbilicus and the hips (Figure 1). The formed plaques changed into atrophic and hyperpigmentation lesions with a porcelain-white center. One year after that, similar plaques appeared on the skin of the legs, affected their lower third part and disseminate circularly around the ankles and the back of the feet (Figure 2). At the same time with the development of the changes of the lower legs, the skin around the wrists also became red and thickened and in the period of one year formed symmetrical scleroatrophic plaques on the flexor surfaces (Figure 3). During the formation of the plaques,

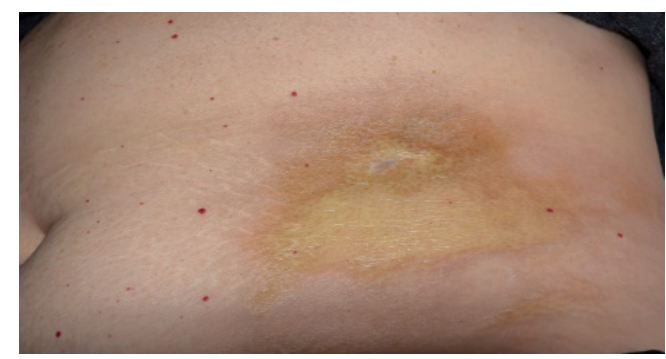

Figure 1. Periumbilical scleroatrophic plaque.

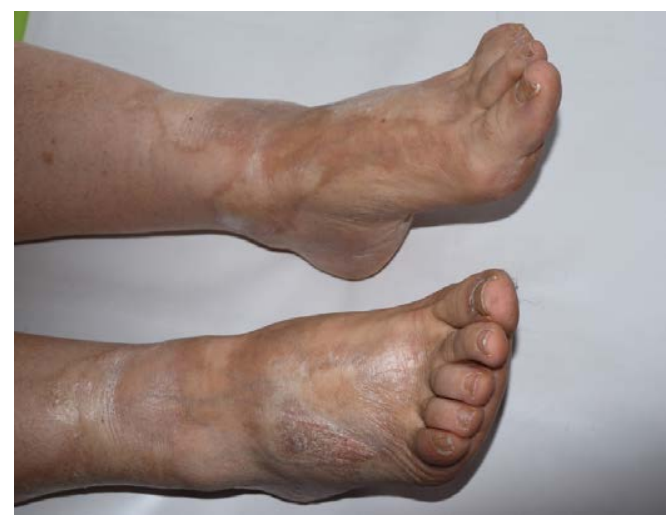

Figure 2. Atrophic plaques with keratotic surface and halo-like pigmentation.

Correspondence to: Stoyan Ivanov Pavlov, Department of Dermatology and Venereology, University Hospital "St.Marina", Medical University of Varna, Varna, Bulgaria, Tel: +359 52978 723; E-mail: stoyanpavlov@abv.bg

Key words: lichen sclerosus, bullous, extragenital lichen sclerosus

Received: June 11, 2017; Accepted: June 28, 2017; Published: June 30, 2017 


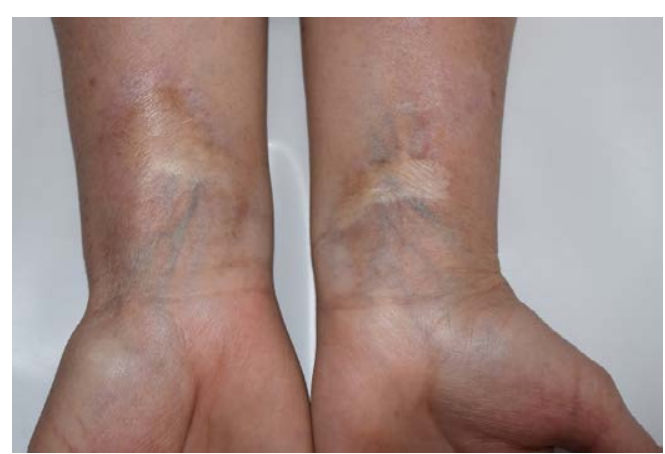

Figure 3. Symmetric whitish atrophic plaques of the flexor surface of the wrists.

the patient complained of moderate burning and discomfort in the affected areas. After the resolution of erythema and the appearance of pigmentation, she complained only of a mild to moderate itching.

The reason for current hospital admission was the emergence of hemorrhagic bulla in the lower pole of the sclero-atrophic plaque on the right lower leg. After the rupture of the blister, ellipsoidal erosion was formed, covered by a hemorrhagic crust (Figure 4).

\section{Objective findings}

The pathological changes affected the limbs and the hips symmetrically. Both of the hips were engaged with round shaped, 5 to $10 \mathrm{~cm}$-sized sclero-atrophic plaques. The skin in the center of the plaques was significantly firm in palpation and there was a pigmented halo in the periphery. Similar sclero-atrofic plaques with irregular shape and sizes (approximately $5 \times 3 \mathrm{~cm}$ ) were located on the flexor surfaces of the wrists. The lower third part of both lower legs was engaged with extensive scleroatrofic plaques, propagated around the ankles and engaging the back of the feet. In the lower pole of oval plaque of almost entirely lower third part of the right leg there was an ellipsoidal flaccid bulla of $4 \times 2 \mathrm{~cm}$ size with hemorrhagic exudate. The edema and erythema on the surrounding tissues were found.

\section{Laboratory and imaging investigations}

The blood count and biochemistry (serum glucose, urea, creatinine, ALT, AST, GGT and coagulation) didn't show abnormalities. The immunological tests found negative antinuclear antibodies, as well as negativity of antibodies to single-stranded DNA and double-stranded DNA and of scleroderma-related panel (antibodies to Sjögren'ssyndrome-related antigen A/Ro, to Scl-70 I antigen and anti-Sjögren's syndrome type B/La). The serology for Lyme disease (IgG and IgM) and antibodies to $\mathrm{HCV}$ were negative. The chest radiology and the abdominal ultrasound were without pathological changes.

\section{Histopathology}

The biopsy was taken from the blister, located on the scleroatrophic plaque of the right lower leg. The pathologist reported for a moderate atrophy and follicular hyperkeratosis of the epidermis with a centrally located big sub-epidermal blood-filled bulla. An increase content of melanin pigments in the basal layer was observed. In the dermis, the collagen bundles were thickened with hyalinization. There was an atrophy of the cutaneous appendages and perivascular lymphocytic infiltrates (Figure 5). The Azan trichrome stain showed: follicular hyperkeratosis in the epidermis; massive hyaline degeneration and fibrosis of connective tissue in the depth dermis and hypodermis; atrophy of hair follicles (Figure 6).

\section{Treatment}

The plaques were locally treated with high-potency topical corticosteroid (Clobetasol propionate $0.05 \%$ cream) for 14 days. A solution with antiseptic action (Povidone-iodine 10\%) was administered for the erosion, caused by a rupture of the bulla. We decided to add to the therapy an intravenous antibiotic (Cefazolin $2 \mathrm{~g}$, three times per day) for 1 week, because of the secondary bacterial infection complicated the rupture of the blister. As a result, the inflammation was reversed and the erosion fully epithelized within a month.

The patient provided written informed consent for planned investigations and use of the results at scientific observations.

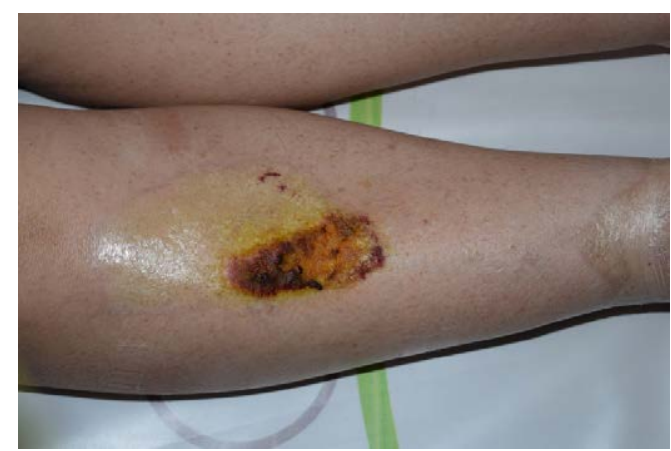

Figure 4. Oval erosion, resulted from rupture of the bulla above the sclero-atrophic plaque.

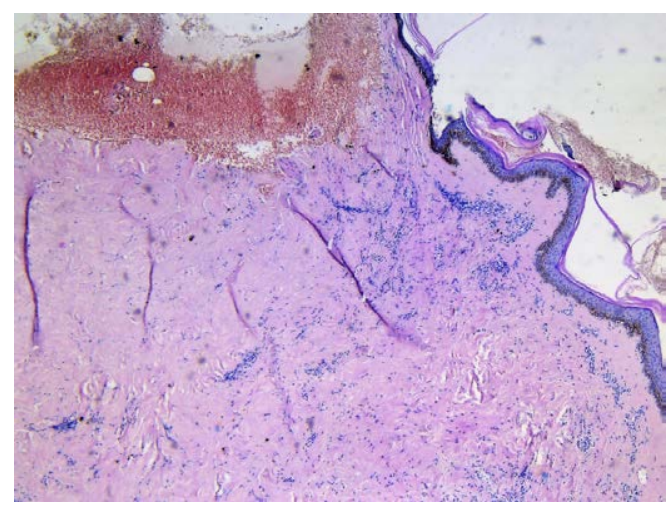

Figure 5. Histology findings of skin biopsy from scleroatrophic plaque of the right lower leg (Hematoxylin-Eosin stain, 25 x 10): sub-epidermal bulla, containing blood.

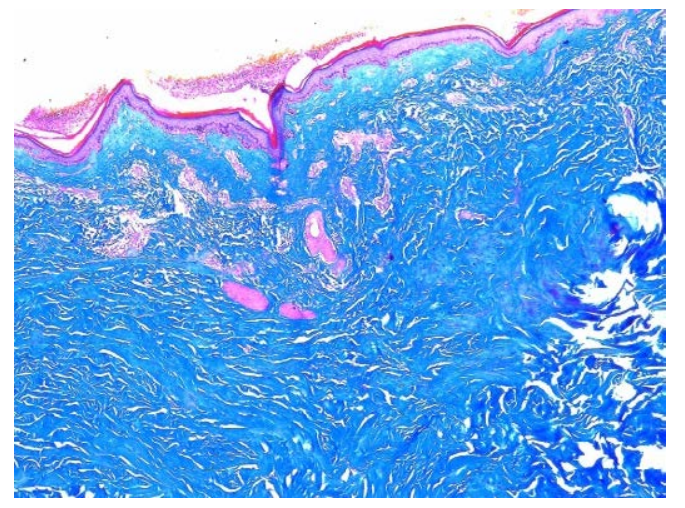

Figure 6. Histology findings of skin biopsy from scleroatrophic plaque of the right lower leg (Azan trichrome stain, $25 \times 10$ ): atrophy and follicular keratosis in the epidermis and sclerosis of the dermis. 


\section{Discussion}

The bullous type of Lichen sclerosus is a rare disease. Lee and colleagues in 2014 [4] found 13 published clinical cases of extragenital bullous LS [4] Kimura et al. [6] established the pathological characteristics of extragenital LS: follicular keratosis and bullous formation.

The etiology of disease isn't clarified. Some authors accepted that Bullous morphea, a rare type of Morphea (Scleroderma circumscripta), are a disease from the same spectrum of LS and described cases of their simultaneous presentation [7]. The association between these diseases of degeneration of connective tissue and sclerosis of the dermis motivated us to look for the presence of serology markers, characteristic for scleroderma in our patient. We tested the antibodies against Scl 70 (topoisomerase 1), which are positive in about $60 \%$ of the patients with systemic sclerosis and the result was negative [8]. Other performed tests also excluded an active autoimmune disease. The negative serology helped to differentiate our case from Lyme disease. We can conclude that based on the characteristic objective findings and histology data for a massive sclerosis in the reticular and papillary dermis, follicular hyperkeratosis and bullous formation the final diagnosis in that case is: Extragenital bullous and hemorrhagic Lichen sclerosus.

The treatment of dermatoses with unclear ethology and pathogenesis is a significant therapeutic challenge. Controversial information about the results of systemic and local therapy of patients with extragenital LS was reported. If the treatment with topical corticosteroids failed to achieve response, systemic corticosteroids, administration of retinoids, chloroquine, tetracycline, then methotrexate or phototherapy must be considered [9]. The recommended local therapy of LS is the application of super-potent topical glucocorticoids (clobethasol propionate), with or without topical calcineurin inhibitors, for long-term daily use. Some evidences support the use of pimecrolimus cream, or intralesion injection of triamcinolone $10 \mathrm{mg} / \mathrm{mL}$ for individual cases $[4,10]$. Firstly, our patient was treated with Cefazolin $6 \mathrm{~g} /$ daily, due to the impetiginization and inflammatory infiltration around the erosion, resulting from the bulla rupture. Then, the treatment continued with local application of Clobethasol propionate on the plaque and local antiseptic (Povidone-iodine 10\%) on the erosion with successful results.

In conclusion, the presented case describes and illustrates a rare bullous-hemorrhagic type of extragenital LS. The satisfactory therapeutic result is achieved by the combination of systemic antibiotic and the local treatment with corticosteroid and antiseptic.

\section{References}

1. Vukicevic J (2016) Extensive bullous lichen sclerosus et atrophicus. An Bras Dermatol 91: 81-83. [Crossref]

2. Lima RS, Maquiné GÁ, Schettini AP, Santos M1 (2015) Bullous and hemorrhagic lichen sclerosus--Case report. An Bras Dermatol 90: 118-120. [Crossref]

3. Rose AE, Boyd KP, Meehan SA, Latkowski JA (2013) Lichen sclerosus et atrophicus. Dermatol Online J 19: 20714. [Crossref]

4. Lee YH, Wu YH (2014) Extragenital bullous lichen sclerosus: A case report and literature review. Dermatologica Sin 32: 169-171.

5. Breier F, Khanakah G, Stanek G, Kunz G, Aberer E, et al. (2001) Isolation and polymerase chain reaction typing of Borrelia afzelii from a skin lesion in a seronegative patient with generalized ulcerating bullous lichen sclerosus et atrophicus. Br J Dermatol 144: 387-392. [Crossref]

6. Kimura A, Kambe N, Satoh T, Togawa Y, Suehiro K, et al. (2011) Follicular keratosis and bullous formation are typical signs of extragenital lichen sclerosus. J Dermatol 38 : 834-836. [Crossref]

7. Yasar S, Mumcuoglu CT, Serdar ZA, Gunes P (2011) A case of lichen sclerosus et atrophicus accompanying bullous morphea. Ann Dermatol 23: S354-S3359. [Crossref]

8. Kuwana M (2017) Circulating Anti-Nuclear Antibodies in Systemic Sclerosis: Utility in Diagnosis and Disease Subsetting. J Nippon Med Sch 84: 56-63. [Crossref]

9. Quatrano NA, Shvartsbeyn M, Meehan SA, Pomerantz R, Pomeranz MK (2015) Extragenital bullous lichen sclerosus. Dermatol Online J: 21. [Crossref]

10. Shiver M, Papasakelariou C, Ahmad Brown J, Wirges M, Kincannon J (2014) Extragenital Bullous Lichen Sclerosus in a Pediatric Patient: A Case Report and Literature Review. Pediatr Dermatol 31: 383-385. [Crossref]

Copyright: (C2017 Pavlov SV. This is an open-access article distributed under the terms of the Creative Commons Attribution License, which permits unrestricted use, distribution, and reproduction in any medium, provided the original author and source are credited. 